Ann Génét. Sél. anim., r979, 11 (4), 39I-396.

\title{
Fréquences de gènes à effets visibles dans la population des chats de la ville de Tours (Indre-et-Loire, France).
}

\author{
Ph. DREUX \\ Laboratoive de Zoologie \\ École Normale Supérieure, 46, rue d'Ulm, 75230 Paris Cedex 05
}

\section{Résumé}

Un échantillon de 220 chats a été examiné à Tours; la population n'a pas montré d'écart à la panmixie. Les fréquences des gènes mutants ont été estimées (tabl. II) et les classes de panachure notées (tabl. III). Les fréquences des allèles $O$ et $a$ y sont particulièrement faibles.

\section{Introduction}

Depuis le premier sondage entrepris par SEARLE en I949 dans la ville de Londres, sur une suggestion de J. B. S. HALDANE, pour estimer la fréquence des gènes mutants dans la population des chats, les tiavaux portant sur ce sujet se sont multipliés, surtout dans les villes. Les résultats de ces études ont montré l'existence de clines dans la fréquence de plusieurs gènes, dont une synthèse provisoire a été faite par TODI en I977. I1 est évident que 1'existence même de ces clines, ainsi que leur signification, ne peut être précisée que par des études aussi nombreuses que possible de façon à dresser une carte exacte des fréquences des gènes intéressants.

Le nombre des sondages effectués en Europe occidentale et sur le pourtour de la Méditerranée dépasse maintenant largement la cinquantaine; il est impossible de les citer tous. Notons seulement ceux qui ont porté sur les effectifs les plus importants et dont les estimations des fréquences géniques sont donc les plus précises :

- Paris (I 200 individus) (DREUX, I967).

- Un district rural de Mayenne (60o individus) (DrEUX, I968). 
- Régions suburbaines du sud de l'Angleterre (400 individus) (RoBINson et Silson, I969).

- Vienne (400 individus) (BAXA, r973).

- La Haye ( 1 ooo individus) (DREUX et LEGEL, I975).

- Marseille (550 individus) (DrEUx, I975).

L'étude de la population de chats présentée dans cet article a été réalisée dans la ville de Tours (Indre-et-Loire).

\section{Méthodes}

Les investigations ont été menées de I973 à I979; l'effectif total de l'échantillon a été de 220 individus. Le refuge de la Société protectrice des animaux a permis d'en examiner 46 . Les 174 autres ont été recensés au cours de quadrillages de la ville, dans les rues, les jardins et les terrains vagues, surtout dans la vieille ville et les faubourgs. Le sexe des animaux était noté chaque fois qu'il a pu être reconnu de façon certaine.

Le polymorphisme de ces chats a été étudié à Tours par rapport aux facteurs génétiques connus, qui portent surtout sur la couleur et la longueur du pelage (RobINSON, I959; SEARLE, I968; Committee on nomenclature, I968) :

- $a$, non agouti, épistatique sur $t$,

$-d$, dilution maltaise,

$-l$, persan : poils longs,

- $O$, orange, lié au sexe, épistatique sur $a$; les femelles hétérozygotes ont le phénotype écaille-de-tortue, où des plages orange se juxtaposent avec des plages normalement colorées,

- $S$, panachure blanche irrégulière, semi-dominant à expressivité variable,

- $t^{b}$, tigré large, allèle récessif de $t^{+}$, tigré ordinaire rayé,

- $W$, blanc dominant, épistatique sur toute les autres couleurs.

L'allèle $T^{a}$ au locus $t$ (tigré type Abyssinie) n'a pas été rencontré dans cet échantillonnage. Trois chats portaient la dilution siamoise $c^{8}$, allèle de la série d'albinisme, récessif par rapport à 1'allèle normal $C$; 1'un d'eux était agouti, indiquant que cet allèle se répand dans la population normale, deux autres étaient des siamois typiques; leur contribution au pool génique de la population est douteux. On a observé 5 individus " chinchilla " (sur phénotype tigré) et 2 "argentés " (sur phénotype non-agouti); le déterminisme de ce facteur considéré classiquement comme allèle du locus $C$ étant douteux, il n'a pas été étudié au point de vue de $1 \mathrm{a}$ fréquence génique. Quant aux anomalies squelettiques, seuls trois chats à queue tordue (kinky-tail) ont été observés, dont un siamois; c'est probablement par l'intermédiaire des siamois de race que cette anomalie (présumée dominante, mais à pénétrance incomplète et expressivité variable) a été introduite dans la population. Aucun individu polydactyle ou syndactyle n'a été repéré, mais, beaucoup d'individus n'ayant pas été examinés de près, cette conclusion n'a rien de certain. 


\section{Résultats}

\section{I. - Généralités}

Les tests statistiques sur plusieurs gènes n'ont pas montré de différence significative entre les chats examinés au refuge de la Société protectrice des animaux et ceux qui ont été vus par quadrillage de la ville. En conséquence, ils ont été réunis en un seul échantillon. Seulement I 8 individus sur 220 étaient visiblement des chatons n'ayant pas atteint la maturité sexuelle (bien que le sexe de beaucoup ait pu être déterminé). En conséquence, il n'était pas possible de faire une étude différentielle valable des jeunes et des adultes.

Aucune femelle ne pouvait être déterminée clairement comme stérilisée, et il n'a été vu que 5 mâles dont la castration était évidente. Il semble bien que la majorité des individus participent effectivement à la reproduction au sein de la population.

Sur tous les chats rencontrés, Ior ont été clairement déterminés comme mâles, 78 comme femelles et le sexe de $4 \mathrm{I}$ d'entre eux n'a pu être reconnu. Le test du $\chi^{2}$ n'est pas significatif et on admettra dans ce qui suit que la proportion des mâles n'est pas statistiquement différente de 50 p. Ioo.

\section{2. - Test de la panmixie sur le gène orange $O$}

Tous les individus examinés peuvent être testés sur les allèles de ce gène, à l'exception des 8 individus (5 mâles et 3 femelles) portant le gène $W$, blanc dominant, épistatique sur tous les autres gènes de coloration; il en restait donc 2 I3 classables pour le gène orange. Les I3 femelles écaille-de-tortue $O /+$ étaient facilement reconnaissables; les 7 individus orange rencontrés étaient tous du sexe mâle.

En admettant que les $2 \times 3$ individus classables étaient également répartis entre les deux sexes (I06 mâles et Io 7 femelles), ils possédaient 320 chromosomes $\mathrm{X}$ dont 20 porteurs du gène orange et $300 \mathrm{du}$ gène sauvage; d'où la fréquence du

\section{TABLEAU I}

Distributions observées et calculées (entre parenthèses)

des phénotypes correspondant au gène orange dans la population des chats de Tours

Observed and expected distribution

of phenotypes corresponding to the orange gene in the cat population of Tours

\begin{tabular}{c|c|c|c}
\hline \hline Génotypes ...... & $O /$ ou $O / O$ & $O /+$ & $+1-$ ou $+1+$ \\
\hline Mâles (ro6) ...... & $7(6,6)$ & 0 & $99(99,4)$ \\
\hline Femelles (107). . . . & $o(0,4)$ & r3 $(\mathrm{I} 2,5)$ & $94(94 \cdot \mathrm{I})$ \\
\hline
\end{tabular}


gène orange, 0,0625 et du gène sauvage 0,9375 . On a calculé sur cette base les fréquences théoriques des différents phénotypes en admettant que la population était panmictique et que le gène $O$ s'y conformait donc à la loi de Hardy-Weinberg. Elles sont présentées dans le tableau $\mathrm{I}$, en comparaison avec les fréquences observées; l'accord s'y révèle excellent (le $\chi^{2}$ est inférieur à I pour 2 degrés de liberté) et on peut admettre l'hypothèse de la panmixie pour le gène $O$ dans l'ensemble de la population.

\section{3. - Fréquences des gènes}

La population des chats de Tours a été trouvée panmictique par rapport au gène orange. Il est donc assez vraisemblable de supposer qu'elle 1'est aussi par rapport aux autres gènes examinés; on calculera donc leurs fréquences par application de la loi de Hardy-Weinberg. Ces fréquences sont données dans le tableau 2 où figure aussi celle de $O$. Les effectifs phénotypiques observés sont ceux de l'homozygote pour les gènes récessifs (exemple : $a a$ ), celui de 1'homozygote ou de 1'hétérozygote pour les gènes dominants (exemple: [W]). Les erreurs absolues maximales pour les fréquences géniques ont été calculées pour un seuil de probabilité de $95 \mathrm{p}$. Ioo. Pour le gène $W$ dont la fréquence est très faible, la distribution n'est pas symétrique autour de la moyenne 0,02 ; la fréquence de $W$ est comprise entre 0,01 et 0,07 .

\section{4. - Panachure}

Tous les chats qui ne présentaient pas le phénotype blanc dominant $(W)$ ont été classés au moyen de l'échelle de panachure déjà définie (DREUX, I967) dans des catégories de o à 0,9 , les nombres exprimant approximativement la fraction du corps de couleur blanche (tab1. 3). On a noté aussi à tout hasard pour des études ultérieures la présence sous la gorge ou sous le ventre (ou les deux) d'une touffe de poils blancs, déjà notée panachure $\varepsilon$; elle était sûrement présente chez I 8 chats au moins qui ont été classés par la suite dans ceux qui ne présentaient pas la panachure irrégulière dont il est question dans ce paragraphe. Le déterminisme de cette panachure discrète est encore mal connu. Au contraire, on sait que la panachure irrégulière est due à un gène semi-dominant $S$, les homozygotes $S S$ étant en moyenne plus panachés de blanc que les hétérozygotes $S s$.

TABLEAU 2

Fréquences des gènes dans la population des chats de Tours

Gene frequencies in the cat population of Tours

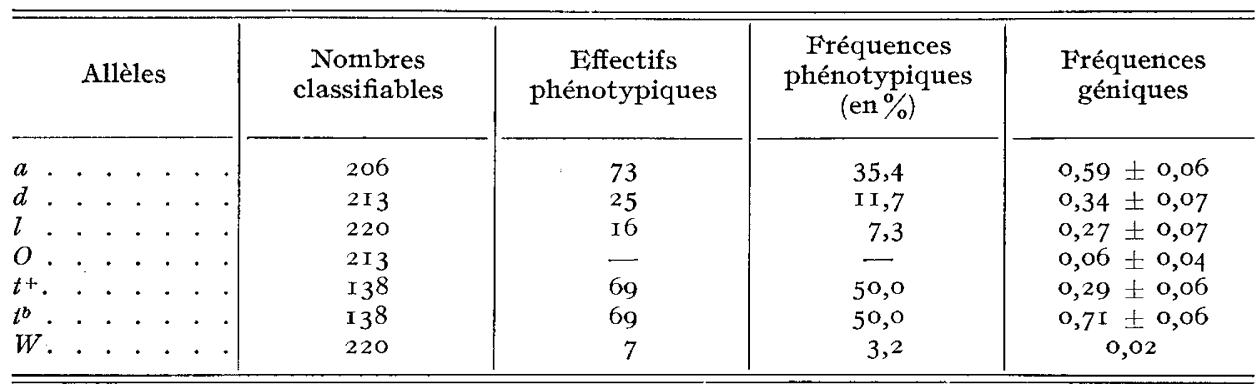


TABLEAU 3

Classes de répartition de la panachure dans la population des chats de Tours Classes for spotting in the cat population of Tours

\begin{tabular}{c|c|c|c|c|c|c|c|c|c|c|c}
\hline Classes de panachure & 0 & $\varepsilon$ & $0, \mathrm{I}$ & 0,2 & 0,3 & 0,4 & 0,5 & 0,6 & 0,7 & 0,8 & 0,9 \\
\hline Effectifs. . . . . . & I I2 & I8 & I2 & I6 & 22 & 8 & 8 & 5 & 7 & 4 & $\mathrm{I}$ \\
\hline
\end{tabular}

La proportion de chats sans panachure irrégulière, donc de génotype ss, permet de calculer la fréquence du gène $S$ dans la population en admettant que s'applique la loi de Hardy-Weinberg. L'allèle $s$ non panaché est homozygote sur $\mathrm{r}_{30}$ individus sur $2 \mathrm{r}_{3}$ classables, il a donc une fréquence de 0,78 et celle de 1'allèle $S$ est par suite de 0,22 , avec des erreurs maximales de 0,05 , calculées comme celles des gènes précédents.

Les valeurs du tableau 3 suggèrent l'hypothèse que les individus dont les repères de panachure sont dans les classes supérieures ou égales à 0,7 sont de génotype $S S$ et que ceux dont le repère de panachure est compris entre $0, I$ et 0,6 sont de génotype $S s$ en application de la loi de Hardy-Weinberg.

\section{Discussion et conclusion}

Cette estimation des fréquences géniques dans une nouvelle ville d'Europe occidentale apporte une preuve supplémentaire du fait que ces fréquences sont proches de 1'équilibre panmictique, du moins pour le locus $O$. Les variations géographiques sont graduelles et peuvent s'exprimer sur la carte par des clines assez réguliers (TODD, I977).

La population de chats de Tours est remarquable à deux égards : la fréquence 0,59 de 1'allèle $a$, non agouti, est la plus basse trouvée jusqu'ici en France; les autres oscillant autour de 0,70 ; et d'autre part, la très basse fréquence $0,06 \mathrm{du}$ gène orange $O$ qui n'a d'équivalent qu'à Paris - o,06 - et à Marseille — o,08 alors que dans les autres régions du globe bien étudiées cette fréquence est en général voisine de 0,20 .

Ces résultats corroborent les cartes de répartition des fréquences géniques de ToDD, qui semblent montrer l'existence d'un couloir nord-sud entre 1'Angleterre et 1'Italie où la fréquence de $O$ est très inférieure et celles de $a$ et $t^{b}$ très supérieures à celles des régions voisines. Cette région a peut-être ioué le rôle, depuis un temps très ancien, de lieu de migration de ces gènes répandant entre autres les allèles $a$ et $t^{b}$ dont 1'origine semble avoir été en Angleterre. L'origine des basses fréquences du gène $O$ est plus douteuse; elles s'étendent en tout cas jusque vers Vienne, en passant par 1'Italie.

Beaucoup de sondages seront encore nécessaires pour préciser ces clines et essayer de les interpréter en termes de génétique écologique. Plusieurs autres sont en cours à l'heure actuelle. 


\section{Summary \\ Gene frequencies in the cat population of Tours}

A sampling of 220 cats of Tours was made; the population was found to be randomly mated. Mutant gene frequencies were estimated (Table II) and white spotting was classified (table III). The frequencies of $O$ and $a$ alleles were found to be very low in the sampling.

\section{Références bibliographiques}

BAXA, H., 1973. Gene frequencies in stray cat populations from Vienna. Genetica, 44, 25-30. COMMITTEE ON STANDARDIZED GENETIC NOMENCLATURE FOR CATS, I968. Standardized genetic nomenclature for the domestic cat. J. Hered., 59, 39-40.

DreuX, Ph., I967. Gene frequencies in the cat population of Paris. J. Hered., 58, 89-92.

DREUX $\mathrm{Ph}$., I968. Gene frequencies in the cat population of a french rural district. J. Hered., $59,37-39$.

Dreux Ph., I975. Génétique de populations des chats domestiques de Marseille (Bouches du Rhône, France). Ann. Génét. Sél. anim., 7, 23-33.

DREUX Ph., LEGEL, J. C., I973. Gene frequencies in the cat population of the Hague. J. Hered., 64, 337-339.

Robinson R., I959. Genetics of the domestic cat. Biblio. Genet., 8, 273-362.

Robinson R., SiLSON M., 1969. Mutant gene frequencies in cats of southern England.Theor. Appl. Genet., 39, 326-329.

SEARLE A. G., I949. Gene frequencies in London's cats. J. Genet., 49, 214-220.

SEARLE A. G., I968. Comparative genetics of coat colour in Mammals. Logos Press/Academic Press, 308 p., New York and London.

Todp N. B., 1977. Cats and commerce. Scient. Amer., 237 (5), 100-107. 